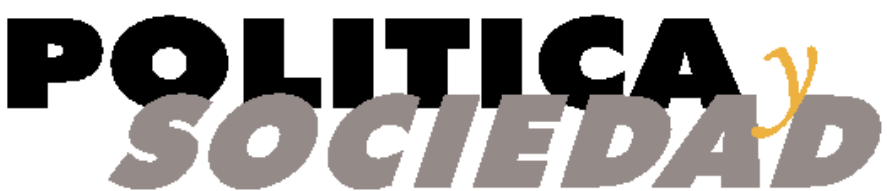

Revista cuatrimestral de Ciencias Sociales

Facultad de Ciencias Políticas y Sociología. Universidad Complutense de Madrid

Presidente

Heriberto Cairo Carou

Director (en funciones)

Laureano Pérez Latorre

Consejo de Redacción

Víctor Abreu Fernández, Luis E. Alonso, Maa Isabel Castaño García, Juan José Castillo Alonso, María Cátedra Tomás, Eduardo Crespo Suárez, Rafael Cruz Martínez Irene Delgado Sotillos, Elena García Gaitán, Montserrat Guibernau, Jesús Leal Maldonado, Omar de León Naveiro, Enrique Luque Baena, Lorenzo Navarrete Moreno, Blanca Olías de Lima Gete, Laureano Pérez Latorre, Josep $\mathrm{M}^{\mathrm{a}} \mathrm{R}$ Reniu, Julio Rodríguez Aramberri, Paloma Román Marugán, Francisco Serra Giménez, Constanza Tobío Soler

\title{
Secretaria
}

Carmen Pérez Hernando

\section{Política local}

\section{Vol. 47 - N. ${ }^{\circ} 3$ (septiembre-diciembre 2010)}

\section{ARTÍCULOS}

Irene Delgado Sotillos

Elecciones municipales en España. Dimensiones analíticas $y$ aspectos distintivos

de ocho procesos electorales (1979-2007)

Guillermo Márquez Cruz Gobernabilidad local en España José Manuel Rodríguez Álvarez

Estructura institucional y organización territorial local en España: fragmentación municipal, asociacionismo confuso,

grandes ciudades y provincias supervivientes

José Manuel Ruano de la Fuente

Contra la participación: discurso y realidad

de las experiencias de participación ciudadana

Gema Pastor Albadalejo y $\mathbf{M}^{\mathrm{a}}$ José García Solana

El Proyecto Municip@

Christian Lefèvre

¿Metrópolis ingobernables? Experiencias europeas

Linze Schaap, Caster Geurtz, Laurens de Graaf

$$
\text { y Niels Karsten }
$$

Innovaciones en los gobiernos subnacionales europeos

\section{Enrique Cabrero Mendoza}

Gobierno y política local en México: luces y sombras de las reformas descentralizadoras
Valdemir Pires y Antonio Carlos Gaeta

El gobierno de las megalópolis, el caso de Brasil - São Paulo: gestión metropolitana, descentralización y participación como desafíos simultáneos

Gerry Stoker

El estudio comparado de la gobernanza local: hacia un enfoque global

\section{VARIOS}

Silvia Marcu

20 años después. Nuevos valores en la sociedad civil de la Rumanía postcomunista

Alejandro $\mathrm{H}$. del Valle

Informalidad y modelos de bienestar en América Latina

\section{RESEÑAS}

\section{Felipe Alpízar}

Pobreza, mujeres y medioambiente de I. Abella, P. Román,

J. Ferri, S. Menéndez-Morán y K. Priego

\section{Manuel Espinel Vallejo}

La mirada social. Un marco de pensamiento distinto para el s. XXI de Alain Touraine

\section{Javier Brines Albiñana}

Estudios sobre la modernización de la Administración Local: Teoría y práctica de El Consultor de los Ayuntamientos y de los Juzgados

\begin{tabular}{|llllll}
\hline & \multicolumn{3}{c|}{ SUSCRIPCIONES } \\
ESPAÑA & & \multicolumn{1}{c|}{ EUROPA } & & \multicolumn{2}{c|}{ RESTO DEL MUNDO } \\
Suscripción individual: & $27.00 €$ & Suscripción individual: & $42.00 €$ & Suscripción individual: & $46.00 €$ \\
Suscripción institucional: & $33.00 €$ & Suscripción institucional: & $48.00 €$ & Suscripción institucional: & $52.00 €$ \\
Número suelto: & $15.00 €$ & Número suelto: & $20.00 €$ & Número suelto: & $24.00 €$ \\
\hline
\end{tabular}




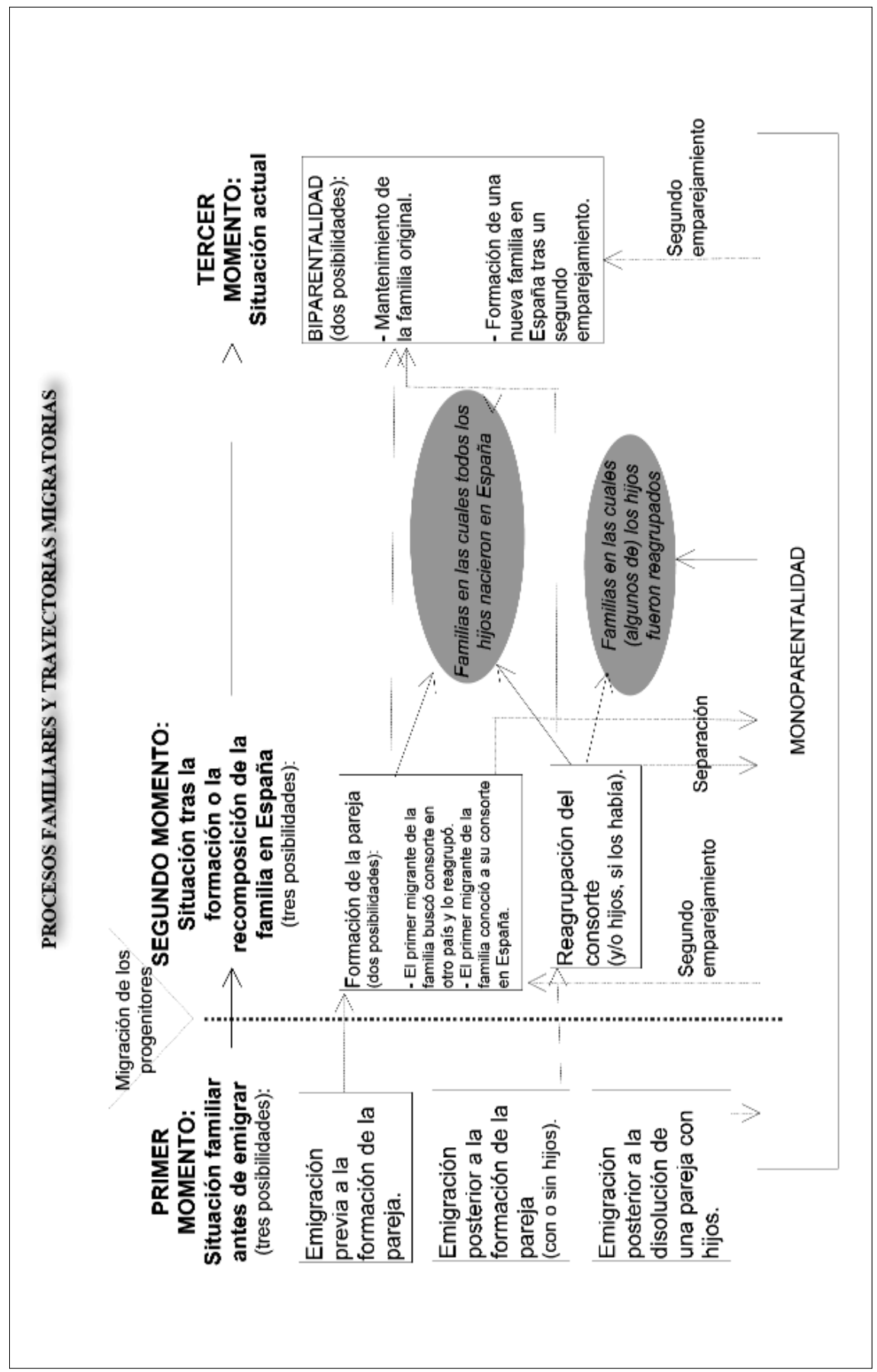




\title{
REVIST'A DE \\ 88 ESTUDIOS \\ 88 REGIONALES
}

\section{$2^{\mathrm{a}}$ EPOCA Mayo-Agosto 2010}

\section{SUMARIO}

\author{
I. Artículos \\ M. Alejandro Cardenete \\ Patricia D. Fuentes Saguar \\ Clemente Polo \\ Montserrat Hermosín Álvarez \\ J. Aníbal Núñez Carrasco \\ Jordi Gallego-Ayala \\ José A. Gómez-Limón \\ Juan Ignacio Pulido Fernández \\ Lucía Isabel García Cebrián \\ Antonio Muñoz Porcar \\ Jean-Baptiste Harguindéguy \\ Pilar Rodríguez \\ Yolanda Ramírez Córcoles \\ Manuel Zafra Víctor \\ Sectores clave de la economía andaluza a partir de la matriz de contabilidad \\ social regional para el año 200 \\ La situaión del impuesto sobre sucesiones y donaciones como tributo cedido \\ a las Comunidades Autónomas. Ejercicio 2010 \\ Un contraste directo de la hipótesis de renta permanente. Evidencia con datos \\ de las Comunidades Autónomas Españolas \\ Escenarios de futuro y sus repercusiones sobre la agricultura de regadío: \\ El caso de la zona regable de Arévalo-Madrigal (Ávila)

\section{Notas} \\ Las partes interesadas en la gestión turística de los parques naturales \\ andaluces. Identificación de interrelaciones e intereses \\ Potencial de las comarcas aragonesas para la localización industrial. \\ Implicaciones para las autoridades públicas \\ Gobernanza multi-nivel y política regional europea. La implementacion de \\ INTERREG III-A España-Francia \\ Medición y gestión del capital intelectual en el ámbito territorial

\section{Documentación} \\ Ley de Autonomía Local de Andalucía
}

Edita: REVISTA DE ESTUDIOS REGIONALES. UNIVERSIDADES DE ANDALUCÍA Secretaría: Facultad de Ciencias Económicas y Empresariales. Universidad de Málaga

El Ejido, s/n. Apartado Oficial Suc. 4 • 29071 Málaga • Telf. 952131297

E-mail: RER@ uma.es / Internet: http://www.revistaestudiosregionales.com 


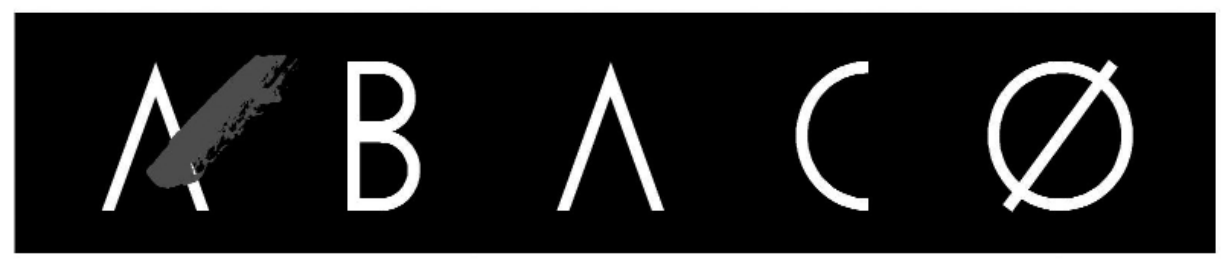

REVISTA DE CULTURA Y CIENCIAS SOCIALES 2010 • NÚMEROS 64/65

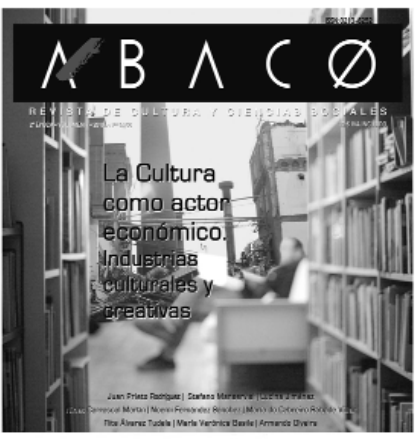

La Cultura como actor económico. Industrias culturales y creativas

ÁBACO, revista de cultura y ciencias sociales, es una iniciativa que surgió en 1986 auspiciada por el Centro de Iniciativas Culturales y Sociales (CICEES), entidad no lucrativa, que concita actividades de un buen número de personas vinculadas al mundo profesional, social y cultural.

Esta publicación trimestral nace con vocación no exclusivamente local, al contrario, pretende incorporar un horizonte amplio, universal.

El contenido de la revista coincide habitualmente en un tema monográfico tratado desde diferentes perspectivas o disciplinas: economía, sociología, psicología, fenómeno cultural, historia, política.

Suscripción personal: $\mathbf{3 0 €}$

Suscripción para instituciones y bibliotecas: $45 €$

Suscripción internacional - Europa: $60 €$ (incluye gastos de envío)

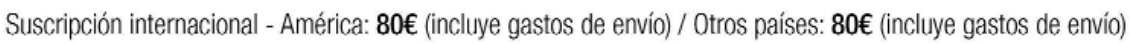

C/ La Muralla, 3 entlo. 33202 GlJóN

Apartado de Correos 202

Telf. y Fax $00+34985319385$

revabaco@arrakis.es_revabaco@telecable.es

\section{¡VISITE NUESTRA WEB!}

En ella podrás leer la versión digital de ÁBACO con artículos exclusivos, suscribirte a la edición en papel, consultar nuestra biblioteca, encontrar enlaces interesantes..., y mucho más. 